\title{
NOTE ON THE PAPER BY MR. F. N. HAWARD ON "THE ORIGIN OF THE ROSTRO-CARINATE INDUSTRY."
}

By Professor Alfred S. Barnes.

Read at Norwich, January 19th, 1920.

Section II. of Mr. Haward's paper deals with the character istics of the fracture and chipping of flint by man and Nature. This section contains so many statements which are incorrect that in the interests of accuracy they cannot be allowed to pass without correction.

The author defines percussion in the following terms:"Sharp, hammer-like rebounding blows impinged (sic) on a flat surface or edge held by a resilient support." If by this definition Mr. Haward intends to convey the impression that in order to flake flint by percussion it is necessary to comply with the conditions he has specified, he is mistaken. It is quite easy, for example, to produce bulbed flakes by percussion under conditions quite different to those set out by Mr. Haward, e.g. by means of a "follow-through" blow on the concave surface of a flint rigidly supported.

On p. 122 of Mr. Haward's paper, we find under the head of Percussion the following :- "Typical cones of percussion struck by Stone Age or Modern man are familiar to all. They are all similar, and are due to a sharp, rebounding, hammer-like blow struck on a flat surface of a flint held by a resilient support. The fracture follows the natural angle of fracture of the material, which is $110^{\circ}$ to $112^{\circ}$ for flint."

In the first place the statement that all cones of percussion produced in the way described are similar, is incorrect. The profile of a cone may be convex, or concave to a varying degree, or may be that of a true cone. Further individual cones are rarely symmetrical and may be convex at one part of the conical surface and concave at another. Neither a flat surface, nor a sharp rebounding blow, nor a resilient support is necessary for the production of cones by percussion.

The vertical angles of flint cones of percuesion varies, so far as my observations go, from about $90^{\circ}$ to $130^{\circ}$, and not between $110^{\circ}$ and $112^{\circ}$ as stated by Mr. Haward. Lastly, the cone of percussion figured by Mr. Haward at " A " in Fig. 25 of this paper cannot be regarded as an accurate representation of a typical cone ; it is more like a lamp shade with silk attachment.

On p. 124 it is stated that "an iron hammer of course produces finer bulbs than one of stone." In this connection I do not know what the term "finer" is intended to connote, but it is evident from the paragraph at the bottom of the page in question that the author does not understand the mechanism of impact. The paragraph in question is as follows :- "The top of cone being 
no doubt due to the flint being resilient, the harder the blow the deeper the flint would compress and the greater the diameter of the top of the cone." Here Mr. Haward has entirely ignored the fact that the area of the frustrum of the cone is also largely affected by the initial curvature of the surfaces of the hammer and of the flint in question, and by the hardness of the hammer employed.

Page 124 concludes with an incorrect statement, as follows :"For a short distance the conical fracture tends to follow the natural angle of a cone. As the force of the blow lessens, the fracture turns sharply towards the mass of the core, and continues downwards, taking its path along the line of least resistance."

This statement is exactly contrary to fact so far as the intermediate stage of the fracture is concerned; in this stage the line of fracture is inclined away from the mass of the core and not towards it, as stated by Mr. Haward.

With regard to Mr. Haward's views relating to the dimensions of bulbs produced under various conditions, I can say that I have reviewed the measurements of nearly 500 bulbed flakes of which I have records and I cannot concur with Mr. Haward nor can I support his " reasoned opinions" expressed in the tables on pp. 128-129.

Figures $31 \mathrm{~A}$ and $31 \mathrm{~B}$ (p. 137) are intended to illustrate the movement termed by Mr. Haward "chip and slide." These figures are misleading. In diagram 31 A the "stationary" (sic) stone which is shewn at " $\mathrm{Y}$ " with its flat face vertical is shewn in Fig. 31 B rotated through an angle of about $220^{\circ}$. As a matter of fact, after rotating in a clockwise direction through an angle of $90^{\circ}$ from its original position, the flat face of the "chip" stone $\mathrm{Y}$ would be parallel to the flat face of the "slide" stone $\mathrm{X}$, and further rotation of the stone $Y$ would cease; hence the position of this stone shewn in diagram $31 \mathrm{~B}$ would not be attained.

With regard to the characteristics of fracture stated by Mr. Haward to be due to " bending or tension," figured A to D in Plate XII., let him take a cylindrical nodule of flint, such as he has illustrated, and place it upon a hard plane surface. He will find that the wedge-shaped section which he considers to be characteristic of "bending or tension" can be produced by a single blow of a hammer delivered on the upper surface of the nodule. 